\title{
Effect of Empagliflozin on Liver Steatosis and Fibrosis in Patients With Non-Alcoholic Fatty Liver Disease Without Diabetes: A Randomized, Double-Blind, Placebo-Controlled Trial
}

\author{
Hoda Taheri · Mojtaba Malek · Faramarz Ismail-Beigi • \\ Farhad Zamani · Masoudreza Sohrabi · Mohammad Reza babaei • \\ Mohammad E. Khamseh
}

Received: August 14, 2020 / Accepted: September 3, 2020 / Published online: September 25, 2020

(C) The Author(s) 2020

\section{ABSTRACT}

Introduction: Despite the high prevalence of non-alcoholic fatty liver disease (NAFLD) and its associated co-morbidities, no efficient treatment in a high percentage of individuals is available. Beneficial effects of sodium-glucose co-transporter 2 inhibitors on fatty liver have been investigated in people with type 2 diabetes (T2DM). The aim of this study was to explore

H. Taheri · M. E. Khamseh

Endocrine Research Center, Institute of

Endocrinology and Metabolism, Iran University of

Medical Sciences (IUMS), Tehran, Iran

M. Malek ( $\square)$

Research Center for Prevention of Cardiovascular Disease, Institute of Endocrinology and Metabolism, Iran University of Medical Sciences (IUMS), Tehran, Iran

e-mail: malek.m@iums.ac.ir

F. Ismail-Beigi

Department of Medicine, Case Western Reserve University, University Hospitals Cleveland Medical Center, Cleveland, OH 44106, USA

F. Zamani · M. Sohrabi

Gastrointestinal and Liver Disease Research Center, Iran University of Medical Sciences (IUMS), Tehran, Iran

M. Reza babaei

Department of Interventional Radiology, Firouzgar Hospital, Iran University of Medical Sciences

(IUMS), Tehran, Iran the effect of empagliflozin on liver steatosis and fibrosis in patients with NAFLD without T2DM. Methods: In this prospective randomized, double-blind, placebo-controlled clinical trial, participants with NAFLD were randomized to empagliflozin $(10 \mathrm{mg} /$ day $)(n=43)$ or placebo $(n=47)$ for 24 weeks. Hepatic steatosis and fibrosis were assessed using transient elastography to measure the controlled attenuation parameter (CAP) and liver stiffness measurement (LSM). The primary outcome was the change in CAP score at 24 weeks.

Results: There was significant decrease in CAP score in both groups but no significant difference was observed between the two groups $(P=0.396)$. LSM was significantly decreased in the empagliflozin-treated group $(6.03 \pm 1.40$ to $5.33 \pm 1.08 \mathrm{kPa} ; P=0.001$ ), while no change was found in the placebo group. In subgroups analysis of patients with significant steatosis at baseline $(\mathrm{CAP} \geq 302 \mathrm{~dB} / \mathrm{m})$, steatosis significantly improved in the empagliflozin group $(37.2 \%$ vs. $17 \% ; P=0.035)$. There was a significant decrease in the grade of liver fat on visual analysis of ultrasound images, AST, ALT, and fasting insulin levels in the empagliflozin group, while no changes were observed in the placebo group.

Conclusions: Empagliflozin improves liver steatosis and, more importantly, measures of liver fibrosis in patients with NAFLD without T2DM. 
Trial registration: ClinicalTrials.gov identifier, IRCT20190122042450N1.

Keywords: Empagliflozin; Liver fibrosis; Nonalcoholic fatty liver disease; Steatosis; Transient elastography

\section{Key Summary Points}

Despite the high prevalence of nonalcoholic fatty liver disease (NAFLD), and its associated co-morbidities, effective treatment is not yet available.

The aim of present study was to explore the effects of empagliflozin on liver steatosis and fibrosis in patients with NAFLD in the absence of type 2 diabetes (T2DM).

Empagliflozin reduces ALT and AST levels.

Empagliflozin improves liver steatosis and, more importantly, measures of liver fibrosis in patients with NAFLD without T2DM.

Improvement in hepatic steatosis was greatest in patients with significant steatosis at baseline (CAP $\geq 302 \mathrm{~dB} / \mathrm{m}$ ).

\section{DIGITAL FEATURES}

This article is published with digital features to facilitate understanding of the article. To view digital features for this article go to https://doi. org/10.6084/m9.figshare.12907526.

\section{INTRODUCTION}

Non-alcoholic fatty liver disease (NAFLD) is a wide spectrum progressive disease and can progress to hepatic cirrhosis and in some individuals to hepatocellular carcinoma [1]. The prevalence of NAFLD is increasing worldwide, and its prevalence among Iranian adults is about $12.5 \%$ [2]. NAFLD is also considered as an

independent risk factor for cardiovascular events and reduced life expectancy [3]. Considering the prevalence of NAFLD and its associated co-morbidities [4], early and appropriate prevention programs and treatment interventions are needed. Although various therapeutic interventions have been reported in recent years, other than sustained weight loss, there are currently no proven therapeutic formats [5].

Sodium-glucose co-transporter 2 (SGLT2) inhibitors increase urinary glucose excretion and lead to a decrease in the blood glucose and insulin levels $[5,6]$. Their use is associated with a significant increase in fatty acid (FA) mobilization from adipose tissues and FA uptake and $\beta$-oxidation in the liver [7]. The beneficial effects of empagliflozin and other SGLT2 inhibitors on liver fat content in patients with T2DM and NAFLD has been reported $[8,9]$.

To the best of our knowledge, no study has been reported on the effect of SGLT2 inhibitors on hepatic fat content in patients with NAFLD who do not have T2DM. Hence, the aim of present study was to explore the effects of empagliflozin on liver steatosis and fibrosis in patients with NAFLD in the absence of T2DM. The selection of this group of patients with NAFLD in the present study was based on the premise that a host of metabolic and other confounding factors that can alter the response of the fatty liver disease to any proposed treatment would be greatly decreased in the absence of concomitant T2DM.

\section{METHODS}

\section{Study Design and Participants}

This was a prospective, randomized, doubleblind, placebo-controlled, clinical trial that included patients with NAFLD but without T2DM. The trial was conducted in accordance with the principles of the Declaration of Helsinki and was approved by the Ethics Committee of Iran University of Medical Sciences (ethics code IR.IUMS.FMD.REC.1398.464). This trial was registered with ClinicalTrials.gov, number IRCT20190122042450N1. All the participants provided written informed consent before study 
entry. The role of Abidi Pharmaceuticals was limited to supply of the medicines, empagliflozin and placebo. Data management was performed by the Institute of Endocrinology and Metabolism monitoring committee that was blinded to the study arms.

We screened individuals aged 20-65 years, who had NAFLD, on the basis of evidence of hepatic steatosis in previous ultrasound imaging or liver function test. T2DM was excluded on the basis of a fasting plasma glucose (FPG) level $\geq 126 \mathrm{mg} / \mathrm{dL}(7.0 \mathrm{mmol} / \mathrm{L})$ or a hemoglobin A1c (HbA1c) level $\geq 6.5 \%(48 \mathrm{mmol} / \mathrm{mol})$ [10]. Other exclusion criteria were as follows: alcohol consumption greater than $20 \mathrm{~g}$ per day in women or greater than $30 \mathrm{~g}$ in men for at least three consecutive months over the past 5 years; history of acute or chronic liver, biliary, or cirrhotic diseases; heart failure (NYHA class 2-4); renal failure (eGFR $<45 \mathrm{~mL} / \mathrm{min} /$ $1.73 \mathrm{~m}^{2}$ ); taking medications associated with fatty liver such as NSAIDs, amiodarone, tamoxifen, sodium valproate, corticosteroids, methotrexate; using supplements including vitamin $\mathrm{E}$, vitamin $\mathrm{C}$, zinc, and selenium or antioxidant agents over the last 3 months; history of cardiovascular events within the past 3 months; pregnancy or breastfeeding; active cancer or history of cancer treatment over the past 2 years; untreated thyroid disorder; and $\mathrm{BMI}>40 \mathrm{~kg} / \mathrm{m}^{2}$.

\section{Para-Clinical and Imaging Investigations}

Ultrasound was performed in all participants using MyLab (Esaote ${ }^{\mathrm{TM}}$ Class $\mathrm{C}$ with linear probe $7 / 5-12 \mathrm{MHz}$ ). Fibroscan of the liver was performed by FibroScan ${ }^{\circledR} 502$ Touch equipped with both $\mathrm{M}$ and XL probes. Controlled attenuation parameter (CAP) and liver stiffness measurement (LSM) were determined [11]. Participants with CAP score greater than $238 \mathrm{~dB} / \mathrm{m}$ were enrolled in the study. Grade 1 steatosis (S1) was defined as CAP between $238 \mathrm{~dB} / \mathrm{m}$ and $259 \mathrm{~dB} / \mathrm{m}$. CAP score between $260 \mathrm{~dB} / \mathrm{m}$ and $291 \mathrm{~dB} / \mathrm{m}$ was considered as $\mathrm{S} 2$, and $292 \mathrm{~dB} / \mathrm{m}$ or higher was defined as S3 [12,13]. In addition, recent cutoff values were also considered: $302 \mathrm{~dB} / \mathrm{m}, 331 \mathrm{~dB} / \mathrm{m}$, and $337 \mathrm{~dB} / \mathrm{m}$, for $\mathrm{S} 1, \mathrm{~S} 2$, and S3, respectively [11]. Liver fibrosis was also determined. In addition, dual energy $\mathrm{x}$-ray absorptiometry (Hologic Discovery DXA system) was performed for all of the participants. Full-body DXA in supine position was performed for analysis of lean and fat masses: android fat ratio (AFR) and skeletal muscle index (SMI). We used fat-free mass in the upper and lower extremities (kilograms) divided by height squared (meters squared), and the appendicular lean mass (ALM) (grams) divided by total body weight (grams) to calculate the SMI $[14,15]$.

Lipid profile, fasting blood glucose, liver functional tests, serum creatinine (Pars biochemical kits using photometric method), HbA1c (Sebia using capillary method), fasting insulin (Monobind kit, code 5825-300, immunoenzymometric assay "IEMA" method), hepatitis viral markers (hepatitis B via SURASE B-96 kit and hepatitis C via NANBASE C 96 Kit), ANA (EuroIMMUN Kit using immunofluorescence method), thyroid function test (ELISA measurement kit by Pishtaz Medical Company), and complete blood count (Sysmex cell counter device using electric resistance-light scattering method) were measured at baseline. The NAFLD fibrosis risk score, FIB-4 (fibrosis 4), APRI (aspartate aminotransferase to platelet ratio index) [16], and insulin resistant state (HOMA2-IR) were also calculated. The HOMA2-IR index was obtained by the program HOMA Calculator [17]. It should be noted that all measurements including FibroScan, ultrasound, DXA, and the biochemical tests were performed in one medical center by the same physicians who were blinded to the study.

\section{Randomization and Follow-Up}

Eligible subjects were randomly assigned in the two study arms using a block randomization method with block size of 4 . Empagliflozin and placebo were quite similar with identical size, shape, color, and packaging. Participants, researcher, and all persons who collected or analyzed the data were blinded to the study arms. Informed consent was obtained from all participants. Patients in the empagliflozin 
group received $10 \mathrm{mg}$ empagliflozin daily for 24 weeks, and participants in the placebo group received placebo tablets for the same period of time. Patients who did not take at least $80 \%$ of the tablets during the study were excluded. Participants were encouraged to perform moderate intensity physical activity in the form of 3-6 times the metabolic equivalent task (METs) for more than three times a week, and they were encouraged to follow the recommendation at least $45 \mathrm{~min}$ without interruption during the study period. Participants were given standard dietary advice as well.

Follow-up included monthly phone calls to assess adherence and possible adverse events including occurrence of genital and urinary tract infections, hypoglycemia, and drug intolerance. All patients had an in-clinic visit 3 months after enrollment. In addition, physical activity was assessed using International Physical Activity Questionnaire (IPAQ) long form [18] at months 0,3 , and 6. A 3-day food frequency questionnaire was used to assess diet regimen at the same intervals. Transient elastography, DXA, and ultrasound images were repeated at 6 months (study end), with the same device and method and by the same physician.

\section{Outcomes}

The primary endpoint was the change in CAP from baseline to 24 weeks of treatment. The key secondary endpoint was the change in LS from baseline to 24 weeks of treatment; other secondary endpoints were the changes in liver enzymes (AST, ALT), fasting insulin, HOMA2, grade of fatty liver by ultrasound, visceral adipose tissue (VAT), and other DXA parameters, and various laboratory scores for hepatic fibrosis.

\section{Statistical Analysis}

Comparison of discrete covariates between the groups, and the before/after measurements were done using the chi-squared test. For continuous variables, paired $t$ test was used to compare before/after measurements. Independent $t$ test was used to compare continuous variables between the groups. Finally, the linear regression models were fitted to assess the effects of the covariates on the outcomes. All $P$ values presented are two-tailed, and differences were considered statistically significant at $P<0.05$.

We calculated that a sample of 86 patients is required to detect a difference of $25 \mathrm{~dB} / \mathrm{m}$ (SD $30 \mathrm{~dB} / \mathrm{m}$ ) in CAP score, with $90 \%$ power at a significance level of $0.01[19,20]$. Considering a $15 \%$ dropout rate, 100 participants were enrolled in the trial.

\section{RESULTS}

A total of 282 individuals were screened for the inclusion/exclusion criteria. One hundred participants who met the eligibility criteria were randomized to receive empagliflozin $(n=50)$ or placebo $(n=50)$. Finally, 43 individuals in the empagliflozin group and 47 in the placebo group completed the trial. The following analysis is based on individuals who completed the trial.

\section{Characteristics of Study Participants}

At baseline, the two groups did not differ regarding demographic and anthropometric characteristics (Table 1). Moreover, calorie intake, physical activity, and biochemical indices were comparable between the two groups at baseline, and there were no significant changes in calorie intake and physical activity within each group and between the groups during the study.

In the empagliflozin group, weight, BMI, and waist circumference all decreased significantly at the end of the trial $(P=0.003,0.002$ and 0.001 , respectively), while no changes were observed in the placebo group.

After 24 weeks, there was a significant decrease in AST and ALT levels in the empagliflozin group, while no significant changes were observed in the placebo group.

By the end of trial, there was a significant decrease in fasting insulin levels in the empagliflozin group: 16.2 (7.4) $\mathrm{mIU} / \mathrm{L}$ to 14.3 (4.8) 
Table 1 Characteristics of the study participants

\begin{tabular}{|c|c|c|c|c|c|c|c|}
\hline & \multicolumn{3}{|c|}{ Empagliflozin $(n=43)$} & \multicolumn{3}{|c|}{ Placebo $(n=47)$} & \multirow[t]{2}{*}{$P$ value $^{+}$} \\
\hline & Enrollment & EOT $^{*}$ & $P$ value & Enrollment & EOT & $P$ value & \\
\hline Age (years) & $43.8(9.7)$ & & & $44.1(9.3)$ & & & 0.875 \\
\hline Sex (male) & $28(65.1 \%)$ & & & $22(46.8 \%)$ & & & 0.081 \\
\hline Weight $(\mathrm{kg})$ & $86.5(12.2)$ & $84.9(13.7)$ & 0.003 & $85.3(12.9)$ & $85.9(13.3)$ & 0.253 & 0.003 \\
\hline BMI $\left(\mathrm{kg} / \mathrm{m}^{2}\right)$ & $30.5(2.3)$ & $29.9(2.8)$ & 0.002 & $30.7(3.5)$ & $30.9(3.8)$ & 0.201 & 0.001 \\
\hline WC $(\mathrm{cm})$ & $104.9(6.5)$ & $102.3(8.3)$ & 0.001 & $106.0(9.0)$ & $104.7(10.6)$ & 0.070 & 0.181 \\
\hline WHR & $0.975(0.045)$ & $0.971(0.049)$ & 0.363 & $0.971(0.053)$ & $0.960(0.057)$ & 0.061 & 0.393 \\
\hline Statin use (yes) & $5(11.6 \%)$ & & & $6(12.8 \%)$ & & & 0.869 \\
\hline FBS (mg/dl) & $94.0(9.2)$ & $96.5(10.0)$ & 0.160 & $91.4(7.8)$ & $95.3(10.7)$ & 0.023 & 0.543 \\
\hline $\operatorname{ALT}(\mathrm{Ut} / \mathrm{l})$ & $39.1(23.6)$ & $32.3(18.2)$ & 0.007 & $33.4(20.7)$ & $31.8(20.0)$ & 0.545 & 0.151 \\
\hline $\operatorname{AST}(\mathrm{Ut} / \mathrm{l})$ & $25.8(10.2)$ & $22.4(7.3)$ & 0.004 & $24.8(9.3)$ & $23.6(9.3)$ & 0.385 & 0.204 \\
\hline $\begin{array}{l}\text { Fasting insulin (mIU/ } \\
\quad \mathrm{L})\end{array}$ & $16.2(7.4)$ & $14.3(4.8)$ & 0.045 & $15.5(8.5)$ & $15.6(8.8)$ & 0.973 & 0.182 \\
\hline HOMA2-IR & $2.08(0.91)$ & $1.86(0.62)$ & 0.067 & $1.99(1.07)$ & $2.00(1.07)$ & 0.901 & 0.183 \\
\hline $\begin{array}{c}\text { Calorie intake } \\
\text { (kcal/day) }\end{array}$ & $2087.8(477.4)$ & $2085.4(541.5)$ & 0.952 & $1949(462.6)$ & $1950(439.9)$ & 0.977 & 0.949 \\
\hline $\begin{array}{l}\text { Physical activity } \\
\text { MET-min/week }\end{array}$ & $\begin{array}{l}2859.6 \\
\quad(3387.5)\end{array}$ & $\begin{array}{l}2224.7 \\
\quad(2266.1)\end{array}$ & 0.149 & $\begin{array}{l}2883.7 \\
\quad(2747.3)\end{array}$ & $\begin{array}{l}2372.0 \\
\quad(1549.6)\end{array}$ & 0.199 & 0.833 \\
\hline
\end{tabular}

Data are the mean \pm SD for normally distributed parameters or $n(\%)$

$B M I$ body mass index, $W C$ waist circumference, $W H R$ waist to hip ratio, FBS fasting blood sugar, ALT alanine transaminase, $A S T$ aspartate aminotransferase, EOT end of trial

$+P$ value for difference between two groups

$\mathrm{mIU} / \mathrm{L}(P=0.045)$; no significant change was observed in the placebo group (Table 1).

\section{Imaging Assessment of NAFLD}

CAP score was comparable between the two groups at baseline $(306.5 \pm 24.0 \mathrm{~dB} / \mathrm{m}$ in the empagliflozin group vs. $304.6 \pm 27.2 \mathrm{~dB} / \mathrm{m}$ in the placebo group, $P=0.734$ ). There was a significant decrease in CAP score in both groups; however, no significant difference was observed between the two groups $(P=0.396)$. No significant association was found between changes in CAP and gender, age, BMI, waist circumference, physical activity, and calorie intake. We then performed subgroups analysis in 44 patients ( 23 patients in the empagliflozin group and 21 in the placebo group) who had significant steatosis at baseline (defined as $\mathrm{CAP} \geq 302 \mathrm{~dB} / \mathrm{m}$ ); the percentage of patients with improved steatosis was significantly greater in the empagliflozin group (37.2\% vs. $17 \%, P=0.035)$.

LSM was significantly decreased after 24 weeks in the empagliflozin group $(6.03 \pm 1.40 \mathrm{kPa}$ to $5.33 \pm 1.08 \mathrm{kPa}, P=0.001)$, while the slight decrease in the placebo group was not significant $(5.56 \pm 1.05 \mathrm{kPa}$ to $5.35 \pm 0.96 \mathrm{kPa}, P=0.139)$. We found no significant association between changes in LSM and gender, age, BMI, waist circumference, physical activity, and calorie intake. Between the two groups, the difference in fibrosis score 
was significantly greater in the empagliflozin group $(P=0.039)$.

After 24 weeks, there was a significant decrease in grade of fatty liver on visual analysis and grading of ultrasound images (done blindly). In the empagliflozin group $44.2 \%$ had grade 2 fatty liver at baseline, while it decreased to $18.6 \%$ at the end of trial; $P=0.001$. In total, by the end of study, $9.3 \%$ of individuals in the empagliflozin group no longer had fatty liver, while no change was observed in the placebo group (Table 2).

\section{Non-Invasive Scoring Systems}

There were no significant changes in either group in the calculated non-invasive parameters examined including the NAFLD fibrosis score, FIB-4 index, and APRI (Table 3).

\section{Body Composition}

After 24 weeks, there was a significant increase in truncal fat mass area in the placebo group, while it increased somewhat but non-significantly in the empagliflozin group, with the increase in truncal fat mass being significantly higher in the placebo group $(P=0.013)$ (Table 4). There was no significant difference between two groups regarding changes in the VAT content $(P=0.251)$, although VAT increased significantly in the placebo group.

There was a significant decrease in skeletal mass index (corrected for height), SMI, which is equal to appendicular lean mass (ALM) normalized against square of height $\left(\mathrm{ALM} / \mathrm{Ht}^{2}\right)$. Given that the height was constant throughout the study, this finding shows that ALM significantly decreased in both groups, while the changes were not significant between two groups $(P=0.091)$.

Table 2 Imaging assessment of NAFLD

\begin{tabular}{|c|c|c|c|c|c|c|c|}
\hline & \multicolumn{3}{|c|}{ Empagliflozin $\left(n^{\mathfrak{s}}\right)$} & \multicolumn{3}{|l|}{ Placebo $\left(n^{\mathfrak{S}}\right)$} & \multirow[t]{2}{*}{$P$ value $^{+}$} \\
\hline & Enrollment & EOT & $P$ value & Enrollment & EOT & $P$ value & \\
\hline CAP score & $306.5(24.0)$ & $277.7(31.9)$ & 0.001 & $304.6(27.2)$ & $281.2(34.7)$ & 0.001 & 0.396 \\
\hline $\mathrm{S} 1>302 \mathrm{~dB} / \mathrm{m}(\%)$ & 41.9 & 16.3 & 0.010 & 29.8 & 23.4 & 0.001 & 0.035 \\
\hline $\mathrm{S} 2>331 \mathrm{~dB} / \mathrm{m}(\%)$ & 0 & 0 & & 2.1 & 0 & & \\
\hline $\mathrm{S} 3>337 \mathrm{~dB} / \mathrm{m}(\%)$ & 11.6 & 0 & & 12.8 & 4.3 & & \\
\hline$S \geq S 1$ & 53.5 & 16.3 & & 44.7 & 27.7 & & \\
\hline LSM, $\mathrm{kPa}$ & $6.03(1.40)$ & $5.33(1.08)$ & 0.001 & $5.56(1.05)$ & $5.35(0.96)$ & 0.139 & 0.039 \\
\hline Fatty liver grade* & & & 0.001 & & & 0.191 & 0.144 \\
\hline Grade 1 (\%) & 53.5 & 67.4 & & 42.6 & 46.8 & & \\
\hline Grade 2 (\%) & 44.2 & 18.6 & & 46.8 & 51.1 & & \\
\hline Grade 3 (\%) & 2.3 & 4.7 & & 10.6 & 2.3 & & \\
\hline Grade $\geq 1(\%)$ & 100 & 90.7 & & 100 & 100 & & \\
\hline
\end{tabular}

$E O T$ end of trial, CAP controlled attenuation parameter, LSM liver stiffness measurement

$\$$ The number of participants for calculation of CAP score mean, LSM, and grade of fatty liver was 43 and 47 in the empagliflozin and placebo groups, respectively. We did a subgroup analysis in participants based on CAP $\geq 302$. In this analysis the number of participants in the empagliflozin group was 23 , and that in the placebo group was 21

$+P$ value for difference between two groups

*Fatty liver grading by ultrasound 
Table 3 Non-invasive scoring systems in assessment of NAFLD

\begin{tabular}{|c|c|c|c|c|c|c|c|}
\hline & \multicolumn{3}{|c|}{ Empagliflozin $(n=43)$} & \multicolumn{3}{|l|}{ Placebo $(n=47)$} & \multirow[t]{2}{*}{$P$ value ${ }^{+}$} \\
\hline & $\overline{\text { Enrollment }}$ & EOT & $P$ value & Enrollment & EOT & $P$ value & \\
\hline NAFLD fibrosis score ${ }^{a}$ & $\begin{array}{r}-2.92 \\
(1.03)\end{array}$ & $\begin{array}{r}-2.98 \\
(1.08)\end{array}$ & 0.694 & $-2.84(1.37)$ & $\begin{array}{r}-2.92 \\
(1.19)\end{array}$ & 0.418 & 0.874 \\
\hline Low risk: $<-1.455(\%)$ & 93.0 & 93.0 & 1.000 & 87.2 & 93.6 & 0.311 & 0.516 \\
\hline $\begin{array}{l}\text { Intermediate risk (\%): } \\
-1.455 \text { to } 0.675\end{array}$ & 7.0 & 7.0 & & 12.8 & 6.4 & & \\
\hline High risk: $>0.675(\%)$ & 0.0 & 0.0 & & 0.0 & 0.0 & & \\
\hline FIB-4 index ${ }^{b}$ & $\begin{array}{l}0.775 \\
\quad(0.307)\end{array}$ & $\begin{array}{l}0.775 \\
\quad(0.293)\end{array}$ & 0.994 & $0.826(0.393)$ & $\begin{array}{l}0.833 \\
\quad(0.464)\end{array}$ & 0.809 & 0.853 \\
\hline Low risk: $<1.3(\%)$ & 90.7 & 95.3 & 0.241 & 91.5 & 89.4 & 0.624 & 0.386 \\
\hline $\begin{array}{l}\text { Intermediate risk: } 1.3-2.67 \\
(\%)\end{array}$ & 9.3 & 4.7 & & 8.5 & 10.6 & & \\
\hline High risk: $>2.67(\%)$ & 0.0 & 0.0 & & 0.0 & 0.0 & & \\
\hline $\mathrm{APRI}^{\mathrm{c}}$ & $\begin{array}{l}0.309 \\
\quad(0.155)\end{array}$ & $\begin{array}{l}0.284 \\
\quad(0.139)\end{array}$ & 0.062 & $0.302(0.147)$ & $\begin{array}{l}0.301 \\
\quad(0.157)\end{array}$ & 0.918 & 0.304 \\
\hline Low risk: $<0.5(\%)$ & 88.4 & 93.0 & 0.283 & 91.5 & 89.4 & 0.714 & 0.439 \\
\hline $\begin{array}{l}\text { Intermediate risk: } 0.5-1.5 \\
(\%)\end{array}$ & 11.6 & 7.0 & & 8.5 & 10.6 & & \\
\hline High risk: $>1.5(\%)$ & 0.0 & 0.0 & & 0.0 & 0.0 & & \\
\hline
\end{tabular}

$E O T$ end of trial

${ }^{a}$ NAFLD fibrosis score formula: $-1.675+0.037 \times$ age $\quad$ [years $]+0.094 \times \mathrm{BMI} \quad\left[\mathrm{kg} / \mathrm{m}^{2}\right]+1.13 \times$ hyperglycemia/diabetes $[$ yes $=1$, no $=0]+0.99 \times$ AST/ALT ratio $-0.013 \times$ platelet $\left[10^{9} / \mathrm{L}\right]-0.66 \times$ albumin $[\mathrm{g} / \mathrm{dL}]$

${ }^{b}$ FIB-4 formula: (Age [years] $\times$ AST $\left.[\mathrm{U} / \mathrm{L}]\right) /\left(\right.$ platelet $\left[10^{9} / \mathrm{L}\right] \times \sqrt{ }$ ALT $\left.[\mathrm{U} / \mathrm{L}]\right)$

$c$ APRI formula: ((AST/AST upper limit of normal)/platelet $\left.\left[10^{9} / \mathrm{L}\right]\right) \times 100$

$+P$ value for difference between two groups

Furthermore, we found a significant decrease in SMI per weight (ALM/Wt) in both groups, with no significant difference between two groups $(P=0.679)$.

There were no changes in the android/gynoid $(A / G)$ ratio and AFR in either group (Table 4).

\section{Adverse Events}

No major adverse event was reported. Mild fungal vaginal infections were reported in two participants in the empagliflozin group and three participants in the placebo group. Mild allergic reactions were reported in one participant in each group. The conditions subsided after receiving appropriate local treatment.

\section{DISCUSSION}

NAFLD is currently the second leading cause of liver transplantation in the USA [21]. Despite this, there is no effective treatment available yet.

We investigated the effect of empagliflozin by measuring CAP, a new quantitative index of hepatic steatosis [11, 12]. Recent studies have 
Table 4 Body composition assessment

\begin{tabular}{|c|c|c|c|c|c|c|c|}
\hline & \multicolumn{3}{|c|}{ Empagliflozin $(n=43)$} & \multicolumn{3}{|c|}{ Placebo $(n=47)$} & \multirow[t]{2}{*}{$P$ value $^{+}$} \\
\hline & Enrollment & EOT & $P$ value & Enrollment & EOT $^{*}$ & $P$ value & \\
\hline Truncal fat mass & $\begin{array}{l}15,627.9 \\
(2788.4)\end{array}$ & $\begin{array}{l}16,139.1 \\
(3527.5)\end{array}$ & 0.069 & $\begin{array}{l}16,088.0 \\
(3658.3)\end{array}$ & $\begin{array}{l}17,481.9 \\
(3900.3)\end{array}$ & 0.001 & 0.013 \\
\hline VAT area & $168.6(44.8)$ & $172.7(50.0)$ & 0.322 & $165.2(40.6)$ & $175.2(44.0)$ & 0.022 & 0.251 \\
\hline SMI (ALM/height $\left.{ }^{2}\right)$ & $8.15(1.25)$ & $7.61(1.27)$ & 0.001 & $7.77(1.19)$ & $7.39(1.06)$ & 0.001 & 0.091 \\
\hline $\begin{array}{l}\text { Low: } m e n<7 \\
\text { women }<5.4(\%)\end{array}$ & 0.0 & 2.3 & 1.000 & 6.4 & 4.3 & 0.724 & 0.315 \\
\hline $\begin{array}{l}\text { High: men } \geq 7 \\
\text { women } \geq 5.4(\%)\end{array}$ & 100.0 & 97.7 & & 93.6 & 95.7 & & \\
\hline SMI (ALM/weight $)^{\mathrm{a}}$ & $26.7(3.6)$ & $25.5(3.8)$ & 0.001 & $25.5(4.0)$ & $24.1(3.8)$ & 0.001 & 0.679 \\
\hline $\begin{array}{l}\text { Low: } m e n \leq 29 \\
\text { women } \leq 25(\%)\end{array}$ & 62.8 & 83.7 & 0.007 & 70.2 & 95.7 & 0.084 & 0.249 \\
\hline $\begin{array}{l}\text { High: men }>29 \\
\text { women }>25(\%)\end{array}$ & 37.2 & 16.3 & & 29.8 & 4.3 & & \\
\hline$A / G$ ratio $^{\mathrm{b}}$ & $0.65(0.15)$ & $0.65(0.15)$ & 0.462 & $0.61(0.13)$ & $0.60(0.12)$ & 0.158 & 0.776 \\
\hline $\mathrm{AFR}^{\mathrm{c}}$ & $0.095(0.015)$ & $0.095(0.015)$ & 0.903 & $0.092(0.013)$ & $0.092(0.012)$ & 0.783 & 0.901 \\
\hline Tertile 1: (0-0.09) (\%) & 34.9 & 34.9 & 0.214 & 59.6 & 53.2 & 0.071 & 0.179 \\
\hline Tertile 2: $(0.09-0.1)(\%)$ & 25.6 & 18.6 & & 10.6 & 25.5 & & \\
\hline Tertile 3: $(0.1-1.0)$ (\%) & 39.5 & 46.5 & & 29.8 & 21.3 & & \\
\hline
\end{tabular}

$A F R$ android fat ratio, EOT end of trial, $V A T$ visceral adipose tissue, $S M I$ skeletal muscle mass index

a $\mathrm{SMI}$ is calculated as ALM/weight $\times 100$, where ALM (appendicular lean mass) is arms lean mass + legs lean mass

b Ratio of android (kg) divided by gynoid (kg)

c Android fat mass/total fat mass

$+P$ value for difference between two groups

shown that CAP is significantly correlated with both the percentage of steatosis and the grade of steatosis compared to liver biopsy used as the gold standard [11, 12]. Considering conventional cut points for the CAP score $(>238 \mathrm{~dB} /$ $\mathrm{m})$, there was no significant difference between the two groups. However, using the new definition criteria for detection of steatosis by transient elastography [11], CAP $>302 \mathrm{~dB} / \mathrm{m}$, a significant decrease in the percentage of individuals with steatosis in the empagliflozintreated group was observed. This finding suggests that CAP scores with strict criteria could be used to detect any significant changes in the measured outcomes. The E-LIFT Trial used MRIPDFF for liver fat estimation [9]. That study showed that an absolute liver fat reduction by $4.1 \%$ correlated with improved histological steatosis and ballooning degeneration in patients with NAFLD. However, the technique is expensive and time-consuming for implementation in clinical practice [22]. Another recent study showed that luseogliflozin (another SGLT2 inhibitor) significantly reduced liver fat in patients with T2DM and NAFLD; however, the liver-to-spleen attenuation ratio was used to estimate liver fat and this method 
may not be as accurate as needed for quantitation of liver fat content [23].

In our study, no significant association was found between changes in CAP and BMI. Similarly, in another study using dapagliflozin, no significant correlation was found between the change in CAP and the changes in body weight, suggesting that dapagliflozin treatment improved liver dysfunction and steatosis by a mechanism unrelated to reduction body weight [20]. In keeping with this, a study from Japan demonstrated that reduction in fatty liver index after ipragliflozin treatment did not correlate with body weight reduction [24]. Possible explanations include the fact that SGLT2 inhibitors decrease de novo lipogenesis in the liver as a result of decreased insulin and glucose levels, and stimulate hepatic $\beta$-oxidation of fatty acids [8].

We also showed a significant decrease in AST and ALT in the empagliflozin group. This finding was also demonstrated by others that found a statistically significant decrease in serum ALT levels after ipragliflozin and canagliflozin treatment $[24,25]$. Therefore, it seems that the beneficial effect of SGLT2 inhibitors on liver enzymes is independent of glycemia status in NAFLD. However, liver enzymes are surrogate indices and do not necessarily predict liver histology response, and elevated liver enzymes do not correlate with grades of NAFLD in histology $[26,27]$. Hence, reduction and/or normalization of liver enzymes after an intervention do not predict improvement in liver histology [26, 27]. It should be noted that it is hepatic fibrosis that determines the natural course of NAFLD [28]. There are no readily available imaging techniques or biomarkers that can effectively replace the use of biopsy in the assessment of the hallmarks of NAFLD, hepatocyte ballooning, and fibrosis, and few studies have used liver biopsy to monitor the efficacy of SGLT2 inhibitors on progression or regression of NAFLD [21]. Lai et al. reported improvements in histological outcomes (steatosis, fibrosis, and hepatocyte ballooning) in $44 \%$ of patients treated with empagliflozin as measured by paired biopsy samples in a single-arm, open-label study [29].
In our study, we used a less invasive procedure to evaluate liver fibrosis; measurement of LSM by transient elastography was reported to be a suitable and non-invasive method of reliably estimating the severity of liver fibrosis [30]. In current study, LSM was significantly decreased after 24 weeks in the empagliflozin group $(P=0.039)$. In another study, LSM decreased in individuals with T2DM that were treated with dapagliflozin, although the changes were not significant [20]. The mechanisms contributing to reduction of hepatic fibrosis by empagliflozin remain unclear. One possible explanation for its antifibrotic effect is that empagliflozin inhibits the hepatic expression of proinflammatory cytokines such as interleukin6 (IL-6), tumor necrosis factor alpha (TNF $\alpha$ ), and monocyte chemoattractant protein 1 (MCP-1), which are the hallmarks of NAFLD [31]. Thus, inhibition of inflammation in the liver may contribute to inhibition of hepatic fibrosis in patients with NAFLD treated with empagliflozin.

Using the FIB-4 index, a validated marker of liver fibrosis, several studies found no significant effects compared with baseline or use of other glucose-lowering agents [20, 32-34]. Nevertheless, two studies demonstrated a significant reduction in FIB-4 index with SGLT2 inhibitor $[35,36]$. In our study there were no significant changes in non-invasive parameters according to the NAFLD fibrosis score, FIB-4 index, and APRI. The reasons for these discrepancies are uncertain, although the patients' clinical characteristics and durations of followup may have varied among the studies.

We also showed a significant decrease in fasting insulin level in the empagliflozin group, while no changes were observed in the placebo group. Decrease in insulin and glucose levels is reported with SGLT2i inhibitors and may have led to a decrease in synthesis of fatty acid in the liver [8]. However, we found no significant changes in insulin resistance state in either group as estimated by the HOMA2 index. Shimizu et al. showed that dapagliflozin compared with placebo significantly decreased insulin resistance in patients with T2DM and NAFLD [20]. In another study diabetic patients with NAFLD treated with pioglitazone and 
ipragliflozin, insulin resistance significantly decreased in the pioglitazone group, while no changes were observed in the ipragliflozin group and there was no significant difference between the two groups [35].

We also determined the body composition using DXA. This method is capable of measuring the lean mass, focusing only on the limbs, and SMI which is based on the lean mass of the extremities could be a good indicator for the estimation of true skeletal muscle mass. We found a significant decrease in SMI calculated either as ALM $/ \mathrm{Ht}^{2}$ or ALM/Wt. A similar reduction for total lean mass was obtained by DXA in another study using SGLT2i therapy [37]. On the other hand, BMI and weight reduction were significantly greater in our empagliflozin group. So, it can be hypothesized that decrements in SMI and the loss of skeletal muscle may be caused protein catabolism secondary to calorie loss through glycosuria [37]. There is the possibility that the reduction of lean muscle mass may lead to sarcopenia, especially in elderly patients [37]. Interestingly, body composition analyses from previous study indicated that approximately two-thirds of SGLT2 inhibitor-associated weight loss can be attributed to the loss of fat mass and one-third to loss of lean mass [38]. In contrast, another study on the effect of ipragliflozin on NAFLD showed that skeletal muscle mass was not reduced significantly, although there was a significant reduction in body fat mass [39].

To the best of our knowledge, this is the first study that has explored the effect of empagliflozin on liver steatosis and fibrosis in patients with NAFLD in the absence of T2DM. However, there were some limitations; we did not perform liver biopsy as the gold standard method to evaluate the status of NAFLD. In addition, at the time when our study was initiated, conventional CAP cutoffs were used to include eligible participants; however, recent biopsy-based comparative studies introduced higher cutoffs for steatosis detected by transient elastography.

\section{CONCLUSION}

This was the first study to investigate the effects of empagliflozin on hepatic steatosis and fibrosis in patients with NAFLD without T2DM. We demonstrated that empagliflozin improves both steatosis and fibrosis in these patients. Empagliflozin might be an effective treatment of individuals with NAFLD who do not have T2DM.

\section{ACKNOWLEDGMENTS}

We greatly appreciate the contribution of the staff of the Endocrine Research Center at Iran University of Medical Sciences (IUMS). We also thank the participants of the study.

Funding. No funding or sponsorship was received for this study or publication of this article.

Authorship. All named authors meet the International Committee of Medical Journal Editors (ICMJE) criteria for authorship for this article, take responsibility for the integrity of the work as a whole, and have given their approval for this version to be published.

Disclosures. Hoda Taheri, Mojtaba Malek, Faramarz Ismail-Beigi, Farhad Zamani, Masoudreza Sohrabi, Mohammad Reza babaei, and Mohammad E. Khamseh have nothing to disclose.

Compliance with Ethics Guidelines. The study was conducted in accordance with the Helsinki Declaration and approved by the Medical Ethical Committee of Endocrine Research Center at Iran University of Medical Sciences (IUMS) (ethics code IR.IUMS.FMD.REC.1398.464). All the participants provided written informed consent before study entry.

Data Availability. The datasets generated during and/or analyzed during the current study are available from the corresponding author on reasonable request.

Open Access. This article is licensed under a Creative Commons Attribution-NonCommercial 4.0 International License, which permits any non-commercial use, sharing, adaptation, distribution and reproduction in 
any medium or format, as long as you give appropriate credit to the original author(s) and the source, provide a link to the Creative Commons licence, and indicate if changes were made. The images or other third party material in this article are included in the article's Creative Commons licence, unless indicated otherwise in a credit line to the material. If material is not included in the article's Creative Commons licence and your intended use is not permitted by statutory regulation or exceeds the permitted use, you will need to obtain permission directly from the copyright holder. To view a copy of this licence, visit http:// creativecommons.org/licenses/by-nc/4.0/.

\section{REFERENCES}

1. Browning JD, Szczepaniak LS, Dobbins R, et al. Prevalence of hepatic steatosis in an urban population in the United States: impact of ethnicity. Hepatology. 2004;40(6):1387-95.

2. Alavian SM, Mohammad-Alizadeh AH, Esna-Ashari F, Ardalan G, Hajarizadeh B. Non-alcoholic fatty liver disease prevalence among school-aged children and adolescents in Iran and its association with biochemical and anthropometric measures. Liver Int. 2009;29(2):159-63.

3. Targher G, Day CP, Bonora E. Risk of cardiovascular disease in patients with nonalcoholic fatty liver disease. N Engl J Med. 2010;363(14):1341-50.

4. Stefan N, Häring H-U, Cusi K. Non-alcoholic fatty liver disease: causes, diagnosis, cardiometabolic consequences, and treatment strategies. Lancet Diabetes Endocrinol. 2019;7(4):313-24.

5. Jung C-H, Mok J-O. The effects of hypoglycemic agents on non-alcoholic fatty liver disease: focused on sodium-glucose cotransporter 2 inhibitors and glucagon-like peptide-1 receptor agonists. J Obes Metab Syndr. 2019;28(1):18.

6. Kahl S, Gancheva S, Straßburger K, et al. Empagliflozin effectively lowers liver fat content in wellcontrolled type 2 diabetes: a randomized, doubleblind, phase 4, placebo-controlled trial. Diabetes Care. 2020;43(2):298-305.

7. Razavi-Nematollahi L, Ismail-Beigi F. Adverse effects of glycemia-lowering medications in type 2 diabetes. Curr Diab Rep. 2019;19(11):132.
8. Gharaibeh NE, Rahhal M-N, Rahimi L, Ismail-Beigi F. SGLT-2 inhibitors as promising therapeutics for non-alcoholic fatty liver disease: pathophysiology, clinical outcomes, and future directions. Diabetes Metab Syndr Obes Targets Ther. 2019;12:1001.

9. Kuchay MS, Krishan S, Mishra SK, et al. Effect of empagliflozin on liver fat in patients with type 2 diabetes and nonalcoholic fatty liver disease: a randomized controlled trial (E-LIFT Trial). Diabetes Care. 2018;41(8):1801-8.

10. American Diabetes Association. Classification and diagnosis of diabetes: standards of medical care in diabetes-2020. Diabetes Care. 2020;43(Supplement 1):S14-S31.

11. Eddowes PJ, Sasso M, Allison M, et al. Accuracy of FibroScan controlled attenuation parameter and liver stiffness measurement in assessing steatosis and fibrosis in patients with nonalcoholic fatty liver disease. Gastroenterology. 2019;156(6):1717-30.

12. Sasso $M$, Beaugrand $M$, De Ledinghen $V$, et al. Controlled attenuation parameter (CAP): a novel VCTE $^{\mathrm{TM}}$ guided ultrasonic attenuation measurement for the evaluation of hepatic steatosis: preliminary study and validation in a cohort of patients with chronic liver disease from various causes. Ultrasound Med Biol. 2010;36(11):1825-35.

13. Yen Y-H, Chen J-B, Cheng B-C, et al. Using controlled attenuation parameter combined with ultrasound to survey non-alcoholic fatty liver disease in hemodialysis patients: a prospective cohort study. PLoS One. 2017;12(4):e0176027.

14. Bouchi R, Nakano Y, Ohara N, et al. Clinical relevance of dual-energy X-ray absorptiometry (DXA) as a simultaneous evaluation of fatty liver disease and atherosclerosis in patients with type 2 diabetes. Cardiovasc Diabetol. 2016;15(1):64.

15. Hsing JC, Nguyen MH, Yang B, et al. Associations between body fat, muscle mass, and nonalcoholic fatty liver disease: a population-based study. Hepatol Commun. 2019;3(8):1061-72.

16. Banini BA, Sanyal AJ. Nonalcoholic fatty liver disease: epidemiology, pathogenesis, natural history, diagnosis, and current treatment options. Clin Med Insights Ther. 2016;8:75-84.

17. The Oxford Center for Diabetes, Endocrinology and Metabolism. HOMA2 Calculator. Oxford: Diabetes Trial Unit; 2009.

18. Wanner M, Probst-Hensch N, Kriemler S, Meier F, Autenrieth C, Martin BW. Validation of the long international physical activity questionnaire: influence of age and language region. Prevent Med Rep. 2016;3:250-6. 
19. Lee Y-H, Kim JH, Kim SR, et al. Lobeglitazone, a novel thiazolidinedione, improves non-alcoholic fatty liver disease in type 2 diabetes: its efficacy and predictive factors related to responsiveness. J Korean Med Sci. 2017;32(1):60-9.

20. Shimizu M, Suzuki K, Kato K, et al. Evaluation of the effects of dapagliflozin, a sodium-glucose co-transporter- 2 inhibitor, on hepatic steatosis and fibrosis using transient elastography in patients with type 2 diabetes and non-alcoholic fatty liver disease. Diabetes Obes Metab. 2019;21(2):285-92.

21. Khan RS, Newsome PN. Fat and fibrosis: does empagliflozin impair the progression of nonalcoholic steatohepatitis in patients with type 2 diabetes mellitus? Dig Dis Sci. 2020;65:342-4.

22. Huwart L, Sempoux C, Vicaut E, et al. Magnetic resonance elastography for the noninvasive staging of liver fibrosis. Gastroenterology. 2008;135(1): 32-40.

23. Shibuya T, Fushimi N, Kawai M, et al. Luseogliflozin improves liver fat deposition compared to metformin in type 2 diabetes patients with non-alcoholic fatty liver disease: a prospective randomized controlled pilot study. Diabetes Obes Metab. 2018;20(2):438-42.

24. Takase T, Nakamura A, Miyoshi H, Yamamoto C, Atsumi T. Amelioration of fatty liver index in patients with type 2 diabetes on ipragliflozin: an association with glucose-lowering effects. Endocr J. 2017;64(3):363-7.

25. Seko Y, Sumida Y, Sasaki K, et al. Effects of canagliflozin, an SGLT2 inhibitor, on hepatic function in Japanese patients with type 2 diabetes mellitus: pooled and subgroup analyses of clinical trials. J Gastroenterol. 2018;53(1):140-51.

26. Portillo-Sanchez P, Bril F, Maximos M, et al. High prevalence of nonalcoholic fatty liver disease in patients with type 2 diabetes mellitus and normal plasma aminotransferase levels. J Clin Endocrinol Metab. 2015;100(6):2231-8.

27. Fracanzani AL, Valenti L, Bugianesi E, et al. Risk of severe liver disease in nonalcoholic fatty liver disease with normal aminotransferase levels: a role for insulin resistance and diabetes. Hepatology. 2008;48(3):792-8.

28. Ekstedt M, Hagström H, Nasr P, et al. Fibrosis stage is the strongest predictor for disease-specific mortality in NAFLD after up to 33 years of follow-up. Hepatology. 2015;61(5):1547-54.
29. Lai L-L, Vethakkan SR, Mustapha NRN, Mahadeva S, Chan W-K. Empagliflozin for the treatment of nonalcoholic steatohepatitis in patients with type 2 diabetes mellitus. Dig Dis Sci. 2020;65(2):623-31.

30. Roulot D, Roudot-Thoraval F, Nkontchou G, et al. Concomitant screening for liver fibrosis and steatosis in French type 2 diabetic patients using Fibroscan. Liver Int. 2017;37(12):1897-906.

31. Farrell GC, Larter CZ. Nonalcoholic fatty liver disease: from steatosis to cirrhosis. Hepatology. 2006;43(S1):S99-S112.

32. Leiter L, Forst T, Polidori D, Balis D, Xie J, Sha S. Effect of canagliflozin on liver function tests in patients with type 2 diabetes. Diabetes Metab. 2016;42(1):25-322.

33. Hawley SA, Ford RJ, Smith BK, et al. The $\mathrm{Na}+$ /glucose cotransporter inhibitor canagliflozin activates AMPK by inhibiting mitochondrial function and increasing cellular AMP levels. Diabetes. 2016;65(9):2784-94.

34. Kurinami N, Sugiyama S, Yoshida A, et al. Dapagliflozin significantly reduced liver fat accumulation associated with a decrease in abdominal subcutaneous fat in patients with inadequately controlled type 2 diabetes mellitus. Diabetes Res Clin Pract. 2018;142:254-63.

35. Ito D, Shimizu S, Inoue K, et al. Comparison of ipragliflozin and pioglitazone effects on nonalcoholic fatty liver disease in patients with type 2 diabetes: a randomized, 24-week, open-label, active-controlled trial. Diabetes Care. 2017;40(10):1364-72.

36. Katsuyama $H$, Hakoshima M, Iijima T, Adachi $H$, Yanai H. Effects of sodium-glucose cotransporter 2 inhibitors on hepatic fibrosis in patients with type 2 diabetes: a chart-based analysis. J Endocrinol Metab. 2020;10(1):1-7.

37. Ohta A, Kato $H$, Ishii $\mathrm{S}$, et al. Ipragliflozin, a sodium glucose co-transporter 2 inhibitor, reduces intrahepatic lipid content and abdominal visceral fat volume in patients with type 2 diabetes. Expert Opin Pharmacother. 2017;18(14):1433-8.

38. Blonde L, Stenlöf K, Fung A, Xie J, Canovatchel W, Meininger G. Effects of canagliflozin on body weight and body composition in patients with type 2 diabetes over 104 weeks. Postgrad Med. 2016;128(4):371-80.

39. Miyake T, Yoshida S, Furukawa S, et al. Ipragliflozin ameliorates liver damage in non-alcoholic fatty liver disease. Open Med (Wars). 2018;13(1):402-9. 